\title{
A Note on the Existence of the Location Parameter Estimate of the Three-Parameter Weibull Model Using the Weibull Plot
}

\author{
Chanseok Park (D) \\ Applied Statistics Laboratory, Department of Industrial Engineering, Pusan National University, Busan 46241, Republic of Korea \\ Correspondence should be addressed to Chanseok Park; cp@pusan.ac.kr
}

Received 2 June 2018; Revised 16 July 2018; Accepted 9 August 2018; Published 9 October 2018

Academic Editor: Enrique Onieva

Copyright (C) 2018 Chanseok Park. This is an open access article distributed under the Creative Commons Attribution License, which permits unrestricted use, distribution, and reproduction in any medium, provided the original work is properly cited.

\begin{abstract}
The Weibull model is one of the widely used distributions in reliability engineering. For the parameter estimation of the Weibull model, there are several existing methods. The method of the maximum likelihood estimation among others is preferred because of its attractive statistical properties. However, for the case of the three-parameter Weibull model, the method of the maximum likelihood estimation has several drawbacks. To avoid the drawbacks, the method using the sample correlation from the Weibull plot is recently suggested. In this paper, we provide the justification for using this new method by showing that the location estimate of the three-parameter Weibull model exists in a bounded interval.
\end{abstract}

\section{Introduction}

There are several existing methods for the location parameter estimation of the three-parameter Weibull model. One can use the method of the maximum likelihood estimation (MLE) which is preferred by many statisticians due to its attractive statistical properties. However, it is well known that the MLE method has several drawbacks for the case of the three-parameter Weibull model. For example, the global maximum can reach infinity at the singularity $\mu=$ $\min \left(x_{1}, x_{2}, \ldots, x_{n}\right)$ and this singularity can result in local maxima of the likelihood function when it is numerically computed. For more details, see Barnard [1] and Smith and Naylor [2].

This MLE method has a convergence issue and it can also have an unfeasible value so that the location estimate of the three-parameter Weibull model can be greater than the minimum value of the observations $[3,4]$. Cheng and Amin [5], Cheng and Iles [6], and Liu et al. [7] also pointed out that the likelihood function has the unbounded likelihood problem and the location parameter tends to approach the smallest observation. Huzurbazar [8] also showed that no stationary point can yield a consistent estimator, which results in no local maximum. Thus, whether a global or a local maximum is sought, the MLE is bound to fail.
To avoid the above problems, several authors, including Gumbel [3] and Vogel and Kroll [4], suggest the method of estimating the parameters using an estimate for the minimum drought. However, in order to estimate the location parameter of the three-parameter Weibull model using the methods in Gumbel [3] or Vogel and Kroll [4], one has to use the special tables provided by Gumbel [3] which are available only for limited cases. Sirvanci and Yang [9] also recommend to estimate the location parameter with $x_{(1)}-1 / n$. However, it is reported that the performance of these methods is not satisfactory. For more details, the reader is referred to Park [10]. It should be noted that this Gumbel method is improved by Park [10]. He proposes to estimate only the location parameter using the ordinary Gumbel method and estimate the other shape and scale parameters using the MLE of the two-parameter Weibull model and shows that the parameter estimates are noticeably improved by the proposed method.

Park [10] also proposed a method which maximizes the sample correlation function from the Weibull plot to estimate the location parameter of the three-parameter Weibull model. Comparing the sample correlations, the p-values, and the Anderson-Darling test statistics, he shows that his method outperforms the afore-mentioned existing methods. His method is conceptually easy to understand, simple to use 
and convenient for practitioners. However, the existence of the location estimate is not yet proved.

In this paper, we show that the location parameter estimate of the three-parameter Weibull model should exist in the bounded interval. Thus, unlike the MLE case, the method by Park [10] does not suffer from nonconvergence, singularity, or infeasibility issues when we calculate the location parameter numerically.

\section{Weibull Plot and Correlation Coefficient from the Plot}

In this section, we briefly review the Weibull plot [11] and present the sample correlation coefficient from the Weibull plot. The Weibull distribution has the respective probability density function and cumulative distribution function (CDF)

$$
\begin{aligned}
f(x) & =\frac{\kappa}{\theta}\left(\frac{x}{\theta}\right)^{\kappa-1} e^{-(x / \theta)^{\kappa}} \\
\text { and } F(x) & =1-e^{-(x / \theta)^{\kappa}} .
\end{aligned}
$$

We let $p=F\left(x_{p}\right)$ for convenience. Then we have

$$
\log (1-p)=-\left(\frac{x_{p}}{\theta}\right)^{\kappa} .
$$

It is immediate from (2) that we have

$$
\log \{-\log (1-p)\}=\kappa \log x_{p}-\kappa \log \theta
$$

It is observed that the plot of $\log \{-\log (1-p)\}$ versus $\log x_{p}$ is ideally a straight line with slope $\kappa$ and intercept $-\kappa \log \theta$ if the data are from the Weibull distribution. The widely used Weibull probability paper in engineering reliability is based on this idea.

With real data, we need to estimate $p=F\left(x_{p}\right)$ to draw the Weibull plot. It should be noted that the estimation of $F\left(x_{(i)}\right)$ is often called the plotting position in the statistics literature. Let $x_{(1)}, x_{(2)}, \ldots, x_{(n)}$ be the order statistics from the smallest to the largest. There are several methods of estimating $F\left(x_{(i)}\right)$ in the literature. Let $p_{i}=\widehat{F}\left(x_{(i)}\right)$ be the empirical CDF value at $x_{(i)}$ for convenience. In practice, the plotting positions such as

$$
\begin{aligned}
p_{i}=\frac{i-3 / 8}{n+1 / 4} \quad \text { for } n \leq 10 \\
\text { and } p_{i}=\frac{i-1 / 2}{n} \quad \text { for } n \geq 11
\end{aligned}
$$

are widely used due to Blom [12] and Wilk and Gnanadesikan [13].

The Weibull plot is constructed by plotting $\log \{-\log (1-$ $\left.\left.p_{i}\right)\right\}$ on the vertical axis and $\log x_{(i)}$ on the horizontal axis. It should be noted that the straightness of the Weibull plot can also be used to assess the goodness-of-fit of the Weibull model. See Park [10] along with the weibullness R package by Park [14]. The measure of the straightness in the Weibull plot can be evaluated by calculating the sample correlation coefficient of the paired points,

$$
\left(\log x_{(i)}, \log \left\{-\log \left(1-p_{i}\right)\right\}\right) .
$$

We let $u_{i}=\log x_{(i)}$ and $v_{i}=\log \left\{-\log \left(1-p_{i}\right)\right\}$ for convenience. Then the sample correlation coefficient from the Weibull plot is defined as

$$
R=\frac{\sum_{i=1}^{n}\left(u_{i}-\bar{u}\right)\left(v_{i}-\bar{v}\right)}{\left[\sum_{i=1}^{n}\left(u_{i}-\bar{u}\right)^{2} \cdot \sum_{i=1}^{n}\left(v_{i}-\bar{v}\right)^{2}\right]^{1 / 2}},
$$

where $\bar{u}=\sum u_{i} / n$ and $\bar{v}=\sum v_{i} / n$.

\section{Existence of the Location \\ Parameter Estimate of the Three-Parameter Weibull Model}

In many reliability applications, failures do not occur below a certain limit which is also known as a failure-free life (FFL) parameter in the engineering literature [15]. The threeparameter Weibull model with this FFL parameter has been widely used to describe the reliability of surface-mount assemblies due to wear-out failures, etc. For more details, see Wong [16], Clech et al. [17], Drapella [18], Mitchell et al. [19], and Lam et al. [20].

It is thus reasonable to consider a lower limit to the Weibull model. This Weibull model is called the threeparameter Weibull with its CDF given by

$$
F(x)=1-\exp \left[-\left(\frac{x-\mu}{\theta}\right)^{\kappa}\right]
$$

where $x>\mu$. This lower limit $\mu$ is often called a location parameter.

Replacing $x_{(i)}$ by $x_{(i)}-\mu$ in (6), we can obtain the sample correlation as a function of $\mu$

$$
R(\mu)=\frac{\sum_{i=1}^{n}\left(u_{i}^{*}-\overline{u^{*}}\right)\left(v_{i}-\bar{v}\right)}{\left[\sum_{i=1}^{n}\left(u_{i}^{*}-\overline{u^{*}}\right)^{2} \cdot \sum_{i=1}^{n}\left(v_{i}-\bar{v}\right)^{2}\right]^{1 / 2}},
$$

where $0 \leq \mu<x_{(1)}, u_{i}^{*}=\log \left(x_{(i)}-\mu\right)$ and $\overline{u^{*}}=\sum u_{i}^{*} / n$. For more details, see Section 5 of Park [10].

It is quite reasonable to impose a condition that $0 \leq \mu<$ $x_{(1)}$ for practical applications. Then the estimate of $\mu$ is given by

$$
\widehat{\mu}=\underset{0 \leq \mu<x_{(1)}}{\arg \max } R(\mu) .
$$

Lemma 1. The function $R(\mu)$ has the limit as

$$
\lim _{\mu \longrightarrow x_{(1)}^{-}} R(\mu)=\frac{\bar{v}-v_{1}}{\left[(1-1 / n) \sum_{i=1}^{n}\left(v_{i}-\bar{v}\right)^{2}\right]^{1 / 2}} .
$$

Proof. In the following, we use the Bachmann-Landau's big $O(\cdot)$ notation. See de Bruijin [21] for more details. That is, if $f(\cdot)$ and $g(\cdot)$ are defined on the domain $D$, then $f(x)=$ $O(g(x))$ means that $|f(x)| \leq K|g(x)|$ for all $x \in D$ where $K$ is a constant. 
For convenience, let $\delta=x_{(1)}-\mu$ and then $u_{1}^{*}=\log \delta$. It is easily seen that as $\delta \longrightarrow 0^{+}$(that is, $\mu \longrightarrow x_{(1)}^{-}$), we have

$$
\begin{aligned}
\sum_{i=1}^{n}\left(u_{i}^{*}-\overline{u^{*}}\right)\left(v_{i}-\bar{v}\right) & =\sum_{i=1}^{n} u_{i}^{*}\left(v_{i}-\bar{v}\right) \\
& =u_{1}^{*}\left(v_{i}-\bar{v}\right)+\sum_{i=2}^{n} u_{i}^{*}\left(v_{i}-\bar{v}\right) \\
& =u_{1}^{*}\left(v_{1}-\bar{v}\right)+O(1)
\end{aligned}
$$

and

$$
\begin{aligned}
\sum_{i=1}^{n}\left(u_{i}^{*}-\overline{u^{*}}\right)^{2} & =\sum_{i=1}^{n} u_{i}^{* 2}-\frac{1}{n}\left(\sum_{i=1}^{n} u_{i}^{*}\right)^{2} \\
& =u_{1}^{* 2}+\sum_{i=2}^{n} u_{i}^{* 2}-\frac{1}{n}\left(u_{1}^{*}+\sum_{i=2}^{n} u_{i}^{*}\right)^{2} \\
& =\left(1-\frac{1}{n}\right) u_{1}^{* 2}+u_{1}^{*} \cdot O(1) .
\end{aligned}
$$

It is immediate upon substituting (11) and (12) into (8) that as $\delta \longrightarrow 0^{+}$, we have

$$
\begin{aligned}
& R(\mu) \\
& =\frac{u_{1}^{*}\left(v_{1}-\bar{v}\right)+O(1)}{\left[(1-1 / n) u_{1}^{* 2}+u_{1}^{*} \cdot O(1)\right]^{1 / 2} \cdot\left[\sum_{i=1}^{n}\left(v_{i}-\bar{v}\right)^{2}\right]^{1 / 2}} .
\end{aligned}
$$

We have $u_{1}^{*} \longrightarrow-\infty$ as $\delta \longrightarrow 0^{+}$. Thus, we let $u_{1}^{* *}=-u_{1}^{*}$ for convenience and we then have $u_{1}^{* *} \longrightarrow \infty$ as $\delta \longrightarrow 0^{+}$. Rewriting (13) using $u_{1}^{* *}$, we have

$$
\begin{aligned}
& R(\mu) \\
& =\frac{u_{1}^{* *}\left(\bar{v}-v_{1}\right)+O(1)}{\left[(1-1 / n) u_{1}^{* * 2}+u_{1}^{* *} \cdot O(1)\right]^{1 / 2} \cdot\left[\sum_{i=1}^{n}\left(v_{i}-\bar{v}\right)^{2}\right]^{1 / 2}} .
\end{aligned}
$$

By dividing both the numerator and denominator of (14) by $u_{1}^{* *}$, we have

$$
\begin{aligned}
& R(\mu) \\
& =\frac{\left(v_{1}-\bar{v}\right)+O(1) / u_{1}^{* *}}{\left[(1-1 / n)+O(1) / u_{1}^{* *}\right]^{1 / 2} \cdot\left[\sum_{i=1}^{n}\left(v_{i}-\bar{v}\right)^{2}\right]^{1 / 2}} .
\end{aligned}
$$

When taking the limit of (15) as $u_{1}^{* *} \longrightarrow \infty$ (that is, as $\delta \longrightarrow$ $0^{+}$), we have

$$
\lim _{\delta \rightarrow 0^{+}} R(\mu)=\frac{\bar{v}-v_{1}}{\left[(1-1 / n) \sum_{i=1}^{n}\left(v_{i}-\bar{v}\right)^{2}\right]^{1 / 2}},
$$

which completes the proof.

Lemma 2. As $\delta \longrightarrow 0^{+}$, we have

$$
\begin{aligned}
\frac{d R(\mu)}{d \mu}= & -\sqrt{\frac{n(n-2)^{2}}{n-1}} \cdot \frac{\operatorname{cov}\left(U^{*}, V\right)}{\left[\sum_{i=1}^{n}\left(v_{i}-\bar{v}\right)^{2}\right]^{1 / 2}} \\
& \cdot \frac{1}{\delta \log ^{2} \delta}+o\left(\frac{1}{\delta \log ^{2} \delta}\right),
\end{aligned}
$$

where $\operatorname{cov}\left(U^{*}, V\right)$ is the sample covariance between $U^{*}$ and $V$ and a series of $n$ observations of $U^{*}$ and $V$ is given by $u_{i}^{*}=$ $\log \left(x_{(i)}-\mu\right)$ and $v_{i}=\log \left\{-\log \left(1-p_{i}\right)\right\}$ for $i=1,2, \ldots, n$.

Proof. Differentiating (8) with respect to $\mu$, we have

$$
\frac{d R(\mu)}{d \mu}=\frac{A \cdot B-C \cdot D}{E}
$$

where

$$
\begin{aligned}
& A=\sum_{i=1}^{n}\left(w_{i}^{*}-\overline{w^{*}}\right)\left(v_{i}-\bar{v}\right), \\
& B=\sum_{i=1}^{n}\left(u_{i}^{*}-\overline{u^{*}}\right)^{2}, \\
& C=\sum_{i=1}^{n}\left(u_{i}^{*}-\overline{u^{*}}\right)\left(v_{i}-\bar{v}\right), \\
& D=\sum_{i=1}^{n}\left(u_{i}^{*}-\overline{u^{*}}\right)\left(w_{i}^{*}-\overline{w^{*}}\right), \\
& E=B^{3 / 2} \cdot\left[\sum_{i=1}^{n}\left(v_{i}-\bar{v}\right)^{2}\right]^{1 / 2},
\end{aligned}
$$

and also

$$
u_{i}^{*}=\log \left(x_{(i)}-\mu\right) \text {, }
$$

$$
w_{i}^{*}=\frac{(-1)}{\left(x_{(i)}-\mu\right)} .
$$

Again, we let $\delta=x_{(1)}-\mu$ for convenience so that we have $u_{1}^{*}=\log \delta$ and $w_{1}^{*}=(-1) / \delta$. Then we can rewrite $A, B, C$, and $D$ as a function of $\delta$ as follows:

$$
\begin{aligned}
A(\delta)= & -\left(v_{1}-\bar{v}\right) \frac{1}{\delta}+K_{1}, \\
B(\delta)= & \left(1-\frac{1}{n}\right) \log ^{2} \delta-\frac{2}{n} \sum_{i=2}^{n} u_{i}^{*} \log \delta+K_{2}, \\
C(\delta)= & \left(v_{1}-\bar{v}\right) \log \delta+K_{3}, \\
D(\delta)= & -\left(1-\frac{1}{n}\right) \frac{\log \delta}{\delta}+\frac{1}{n} \sum_{i=2}^{n} u_{i}^{*} \frac{1}{\delta}-\frac{1}{n} \sum_{i=2}^{n} w_{i}^{*} \log \delta \\
& +K_{4},
\end{aligned}
$$

and also

$$
E(\delta)=B(\delta)^{3 / 2} \cdot\left[\sum_{i=1}^{n}\left(v_{i}-\bar{v}\right)^{2}\right]^{1 / 2},
$$

where

$$
\begin{aligned}
& K_{1}=\sum_{i=2}^{n} w_{i}^{*}\left(v_{i}-\bar{v}\right), \\
& K_{2}=\sum_{i=2}^{n} u_{i}^{* 2}-\frac{\left(\sum_{i=2}^{n} u_{i}^{*}\right)^{2}}{n}, \\
& K_{3}=\sum_{i=2}^{n} u_{i}^{*}\left(v_{i}-\bar{v}\right),
\end{aligned}
$$




$$
K_{4}=\sum_{i=2}^{n} u_{i}^{*} w_{i}^{*}-\frac{1}{n}\left(\sum_{i=2}^{n} u_{i}^{*}\right)\left(\sum_{i=2}^{n} w_{i}^{*}\right) .
$$

It should be noted that $K_{1}, K_{2}, K_{3}$, and $K_{4}$ do not include $\delta$. After some tedious algebra, when $\delta \longrightarrow 0^{+}$, we have

$$
\begin{aligned}
A(\delta) \cdot B(\delta)-C(\delta) \cdot D(\delta) & \frac{n-1}{n}\left[\sum_{i=2}^{n} u_{i}^{*} v_{i}^{*}-\frac{1}{n-1} \sum_{i=2}^{n} u_{i}^{*} \sum_{i=2}^{n} v_{i}^{*}\right] \frac{1}{\delta} \log \delta \\
& +O\left(\frac{1}{\delta}\right)+O\left(\log ^{2} \delta\right)+O(\log \delta) .
\end{aligned}
$$

Since $\log \delta<0$ as $\delta \longrightarrow 0^{+}$, we have $\log \delta=-|\log \delta|$. The sample covariance between $U^{*}$ and $V$ is given by

$$
\operatorname{cov}\left(U^{*}, V\right)=\frac{1}{n-2}\left[\sum_{i=2}^{n} u_{i}^{*} v_{i}-\frac{1}{n-1} \sum_{i=2}^{n} u_{i}^{*} \sum_{i=2}^{n} v_{i}\right] .
$$

It is easily shown that

$$
O\left(\frac{1}{\delta}\right)+O\left(\log ^{2} \delta\right)+O(\log \delta)=o\left(\frac{1}{\delta} \log \delta\right)
$$

where $o(\cdot)$ is the Bachmann-Landau's little $o$-notation in de Bruijin [21] for example. That is, $f(x)=o(g(x))$ implies that $f(x) / g(x) \longrightarrow 0$ as $x \longrightarrow c$.

Substituting $\log \delta=-|\log \delta|,(26)$ and (27) into (25), we have

$$
\begin{aligned}
A(\delta) & B(\delta)-C(\delta) \cdot D(\delta) \\
= & -\frac{(n-1)(n-2)}{n} \cdot \operatorname{cov}\left(U^{*}, V\right) \cdot \frac{1}{\delta}|\log \delta| \\
& +o\left(\frac{1}{\delta} \log \delta\right) .
\end{aligned}
$$

Similarly, we can rewrite $E(\delta)$ as

$$
=\left[\left(1-\frac{1}{n}\right) \log ^{2} \delta+O(\log \delta)\right]^{3 / 2}\left[\sum_{i=1}^{n}\left(v_{i}-\bar{v}\right)^{2}\right]^{1 / 2} .
$$

Substituting (28) and (29) into (18), we have

$$
\begin{aligned}
\frac{d R(\mu)}{d \mu} & =-\frac{(n-1)(n-2) / n \cdot \operatorname{cov}\left(U^{*}, V\right) \cdot(1 / \delta)|\log \delta|+o((1 / \delta) \log \delta)}{\left[(1-1 / n) \log ^{2} \delta+O(\log \delta)\right]^{3 / 2}\left[\sum_{i=1}^{n}\left(v_{i}-\bar{v}\right)^{2}\right]^{1 / 2}} \\
& =-\sqrt{\frac{n(n-2)^{2}}{n-1}} \cdot \frac{\operatorname{cov}\left(U^{*}, V\right)}{\left[\sum_{i=1}^{n}\left(v_{i}-\bar{v}\right)^{2}\right]^{1 / 2}} \cdot \frac{1}{\delta \log ^{2} \delta}+o\left(\frac{1}{\delta \log ^{2} \delta}\right),
\end{aligned}
$$

which completes the proof.

Theorem 3. The global maximum of $R(\mu)$ exists on $\left[0, x_{(1)}\right)$ with $n \geq 3$.

Proof. The function $R(\mu)$ is continuous on $\left[0, x_{(1)}\right)$. Considering the result of Lemma 1, we define

$$
R\left(x_{(1)}\right)=\frac{\bar{v}-v_{1}}{\left[(1-1 / n) \sum_{i=1}^{n}\left(v_{i}-\bar{v}\right)^{2}\right]^{1 / 2}} .
$$

Then $R(\mu)$ is continuous on the closed bounded interval $\left[0, x_{(1)}\right]$. Thus, the function $R(\mu)$ has a global maximum and a global minimum on $\left[0, x_{(1)}\right]$, due to Theorem 4.28 in Apostol [22]. Note that $\mu=x_{(1)}$ is a singularity point. Thus, it suffices to show that the global maximum of $R(\mu)$ is not obtained at $\mu=x_{(1)}$.

Since $u_{1}^{*}<u_{2}^{*}<\cdots<u_{n}^{*}$ and $v_{1}<v_{2}<\cdots<v_{n}$, it is easily seen that the term $\operatorname{cov}\left(U^{*}, V\right)$ in Lemma 2 is always positive. It is immediate from the L'Hôpital's rule that we have

$$
\lim _{\delta \rightarrow 0^{+}} \frac{1}{\delta \log ^{2} \delta}=\infty
$$

Thus, using these with Lemma 2, we have

$$
\frac{d R(\mu)}{d \mu} \longrightarrow-\infty
$$

as $\delta \longrightarrow 0^{+}$(that is, as $\mu \longrightarrow x_{(1)}^{-}$). Since $R(\mu)$ is differentiable on $\left(0, x_{(1)}\right)$, it is easily seen that $R\left(x_{(1)}\right)$ cannot be a maximum from the intermediate value property of derivatives. For more details, see Lemma 6.2.11 of Bartle and Sherbert [23]. Thus, the global maximum exists on $\left[0, x_{(1)}\right)$.

It is worthwhile to mention the lower bound of the sample correlation coefficient from the Weibull plot. It is well known that the sample correlation coefficient should be in $[-1,1]$ in general. However, in the Weibull plot, the data and plotting positions are ordered and thus the sample correlation coefficient should be positive. Also, it should be noted that with the order statistics restriction, the sample correlation coefficient is bounded below by $1 /(n-1)$ which is the best lower bound due to Hwang and $\mathrm{Hu}[24]$.

Finally, after the location parameter is obtained, we can estimate the other shape and scale parameters by several existing methods. We recommend the MLE method of the two-parameter Weibull for the estimation of shape and scale. 
TABLE 1: Parameter estimates, correlations, $\mathrm{p}$ value, and log-likelihood under consideration.

\begin{tabular}{lcccccc}
\hline Method & $\widehat{\mu}$ & $\widehat{\kappa}$ & $\widehat{\theta}$ & $R(\widehat{\mu})$ & p-value & log-likelihood \\
\hline Proposed & 139.33 & 1.29174 & 1032.2 & 0.98135 & 0.6066 & -156.4056 \\
McLE & 123.46 & 1.15000 & 1081.7 & 0.98075 & 0.5870 & -156.8671 \\
Min. SSE & 125.23 & 1.15000 & 1081.7 & 0.98086 & 0.5906 & -156.8455 \\
\hline
\end{tabular}
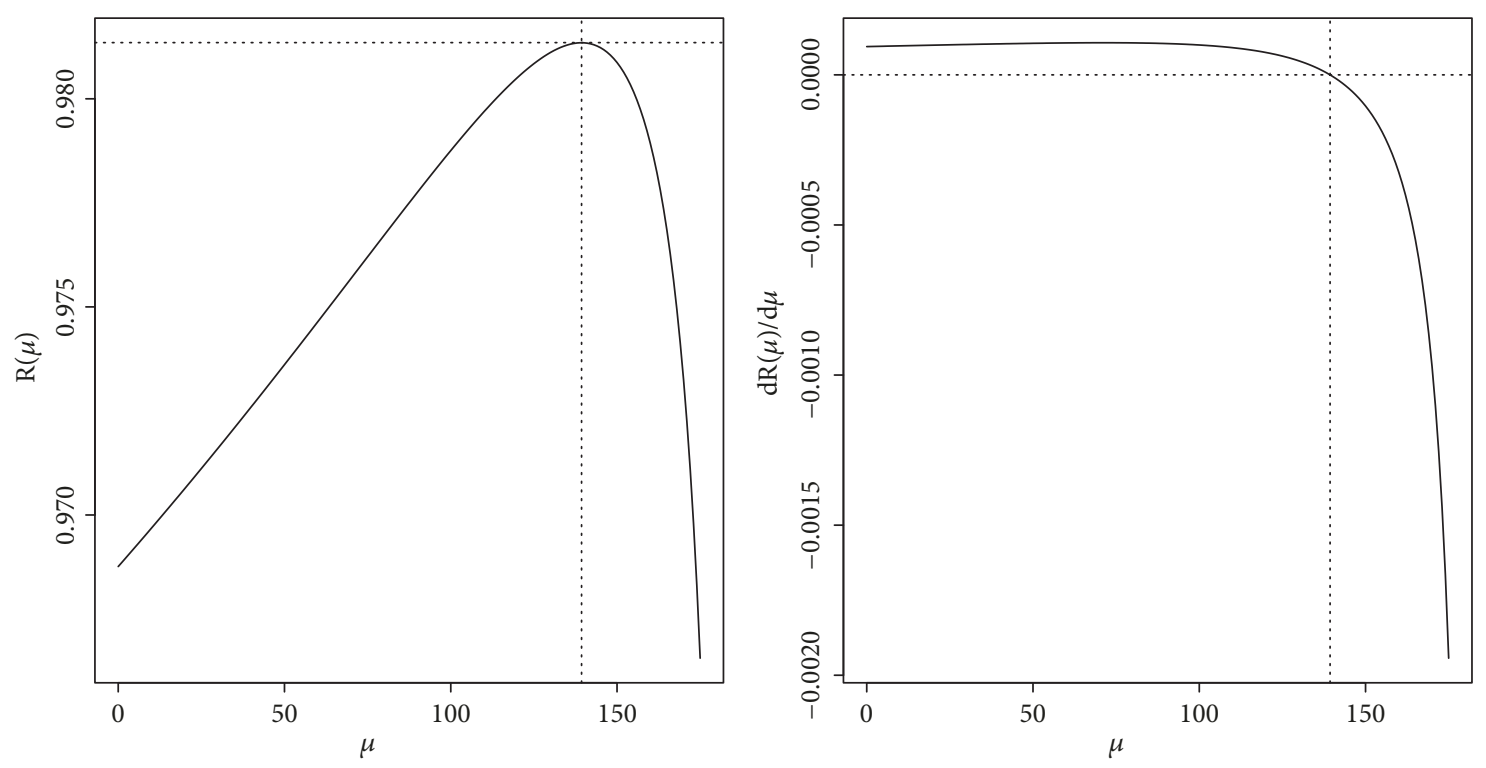

Figure 1: Correlation function, $R(\mu)$, and $d R(\mu) / d \mu$.

For more details, see Section 5 of Park [10]. Unlike the MLE of the three-parameter Weibull, the MLE of the twoparameter Weibull guarantees the existence and uniqueness due to Farnum and Booth [25].

\section{An Illustrative Example}

The data in this example, published in Bilikam et al. [26], are the numbers of miles to failure of a type of vehicle. This data set has since then been often used for illustration of a threeparameter Weibull distribution [6, 20].

We can estimate the location parameter by maximizing the correlation function in (8) or solving $d R(\mu) / d \mu=0$ in (18) as shown in Figure 1 which results in $\widehat{\mu}=139.33$. As recommended earlier, we estimated the other shape and scale parameters using the MLE of the two-parameter Weibull.

In order to examine the performance of the proposed method, we compare it with other existing methods in Lam et al. [20]. They estimated the parameters using the constrained MLE (McLE) approach and the minimum SSE approach. The results are summarized in Table 1 with the corresponding $R(\widehat{\mu}), \mathrm{p}$ value for Weibullness, and log-likelihood. Note that the $\mathrm{p}$ values for Weibullness testing were obtained using the weibullness $\mathrm{R}$ package by Park [14]. The results show that the proposed method outperforms the existing methods.

\section{Data Availability}

The article has no data used for study. So, it belongs to case 12 in the statement of the provided URL: that is, "12. No data were used to support this study".

\section{Conflicts of Interest}

The author declares that they have no conflicts of interest.

\section{Acknowledgments}

This work was supported by the National Research Foundation of Korea (NRF) grant funded by the Korea government (No. NRF-2017R1A2B4004169). The author also wishes to dedicate this work to the memory and honor of Professor Byung Ho Lee in the Department of Nuclear Engineering at Seoul National University. The author's interests in mathematics were formed under the strong influence of Professor Lee who passed away peacefully in Seoul on July 9, 2001.

\section{References}

[1] G. A. Barnard, "The use of the likelihood function in statistical practice," in Proceedings of the Fifth Berkeley Symposium on Mathematical Statistics and Probability, vol. 1, pp. 27-40, University of California Press, Berkeley, Calif, USA, 1965. 
[2] R. L. Smith and J. C. Naylor, "A comparison of maximum likelihood and Bayesian estimators for the three-parameter Weibull distribution," Journal of the Royal Statistical Society: Series C (Applied Statistics), vol. 36, no. 3, pp. 358-369, 1987.

[3] E. Gumbel, "Statistical forecast of droughts," Hydrological Sciences Journal, vol. 8, no. 1, pp. 5-23, 1963.

[4] R. M. Vogel and C. N. Kroll, "Low-flow frequency analysis using probability-plot correlation coefficients," Journal of Water Resources Planning and Management, vol. 115, no. 3, pp. 338-357, 1989.

[5] R. C. Cheng and N. A. Amin, "Estimating parameters in continuous univariate distributions with a shifted origin," Journal of the Royal Statistical Society Series B (Methodological), vol. 45, no. 3, pp. 394-403, 1983.

[6] R. C. Cheng and T. C. Iles, "Embedded models in threeparameter distributions and their estimation," Journal of the Royal Statistical Society Series B (Methodological), vol. 52, no. 1, pp. 135-149, 1990.

[7] S. Liu, H. Wu, and W. Q. Meeker, "Understanding and addressing the unbounded "likelihood" problem," The American Statistician, vol. 69, no. 3, pp. 191-200, 2015.

[8] V.S. Huzurbazar, "The likelihood equation, consistency and the maxima of the likelihood function," Annals of Eugenics, vol. 14, pp. 185-200, 1948.

[9] M. Sirvanci and G. Yang, "Estimation of the weibull parameters under type I censoring," Journal of the American Statistical Association, vol. 79, no. 385, pp. 183-187, 1984.

[10] C. Park, "Weibullness test and parameter estimation of the three-parameter weibull model using the sample correlation coefficient," International Journal of Industrial Engineering : Theory, Applications and Practice, vol. 24, no. 4, pp. 376-391, 2017.

[11] W. Nelson, Applied Life Data Analysis, John Wiley and Sons, New York, NY, USA, 1982.

[12] G. Blom, Statistical Estimates and Transformed Beta-Variables, John Wiley \& Sons, New York, NY, USA, 1958.

[13] M. B. Wilk and R. Gnanadesikan, "Probability plotting methods for the analysis of data," Biometrika, vol. 55, no. 1, pp. 1-17, 1968.

[14] C. Park, Weibullness: Goodness-Of-Fit Test for Weibull Distribution (R Package), 2018, https://cran.r-project.org/web/packages/ weibullness.

[15] Reliability HotWire, Reliability Basics - Location Parameter of The Weibull Distribution, vol. 15, May 2002, http://www.weibull .com/hotwire/issue15/relbasics15.htm.

[16] K. L. Wong, "When reliable means failure free," Quality and Reliability Engineering International, vol. 3, no. 3, pp. 145-145, 1987.

[17] J.-P. M. Clech, D. M. Noctor, J. C. Manock, G. W. Lynott, and F. E. Bader, "Surface mount assembly failure statistics and failure free time," in Proceedings of the 1994 IEEE 44th Electronic Components \& Technology Conference, pp. 487-497, May 1994.

[18] A. Drapella, "An improved failure-free time estimation method," Quality and Reliability Engineering International, vol. 15, no. 3, pp. 235-238, 1999.

[19] D. Mitchell, B. Zahn, and F. Carson, "Board level thermal cycle reliability and solder joint fatigue life predictions of multiple Stacked Die chip scale package configurations," in Proceedings of the 54th Electronic Components and Technology Conference, pp. 699-703, USA, June 2004.

[20] S.-W. Lam, T. Halim, and K. Muthusamy, "Models with failurefree life-applied review and extensions," IEEE Transactions on Device and Materials Reliability, vol. 10, no. 2, pp. 263-270, 2010.
[21] N. G. de Bruijn, Asymptotic Methods in Analysis, Dover Publications, New York, NY, USA, 3rd edition, 1981.

[22] T. M. Apostol, Mathematical Analysis, Addison-Wesley Publication, Reading, Mass, USA, 2nd edition, 1974.

[23] R. G. Bartle and D. R. Sherbert, Introduction to Real Analysis, John Wiley and Sons, New York, NY, USA, 4th edition, 1982.

[24] T. Y. Hwang and C. Y. Hu, "The best lower bound of sample correlation coefficient with ordered restriction," Statistics and Probability Letters, vol. 19, no. 3, pp. 195-198, 1994.

[25] N. R. Farnum and P. Booth, "Uniqueness of maximum likelihood estimators of the 2-parameter weibull distribution," IEEE Transactions on Reliability, vol. 46, no. 4, pp. 523-525, 1997.

[26] J. E. Bilikam, "k-sample maximum likelihood ratio test for change of weibull shape parameter," IEEE Transactions on Reliability, vol. R-28, no. 1, pp. 47-50, 1979. 


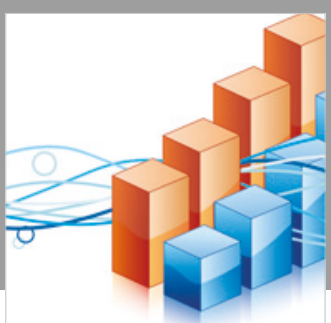

Advances in

Operations Research

\section{-n-m}
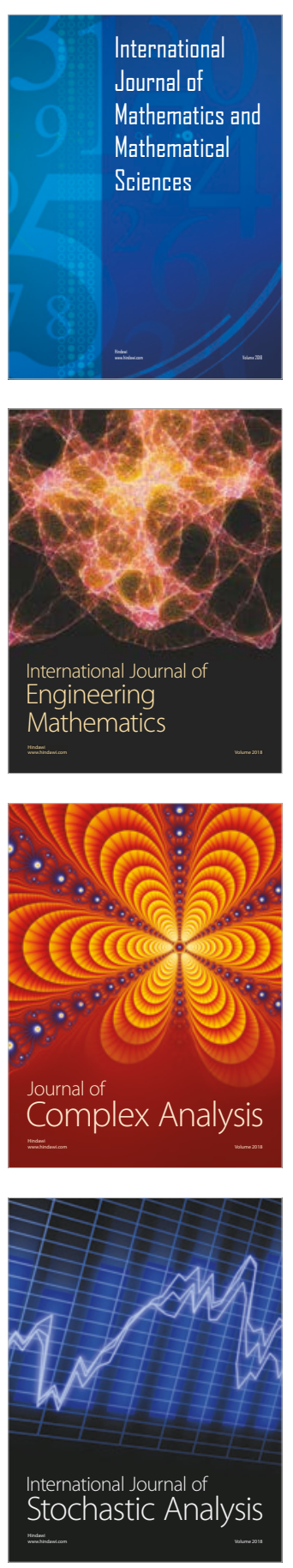
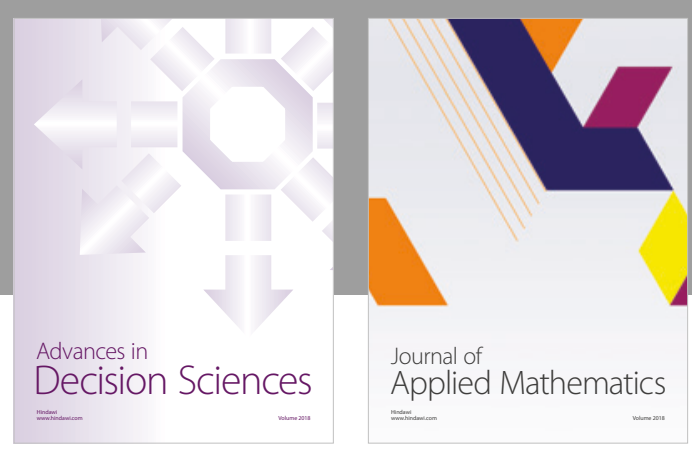

Journal of

Applied Mathematics
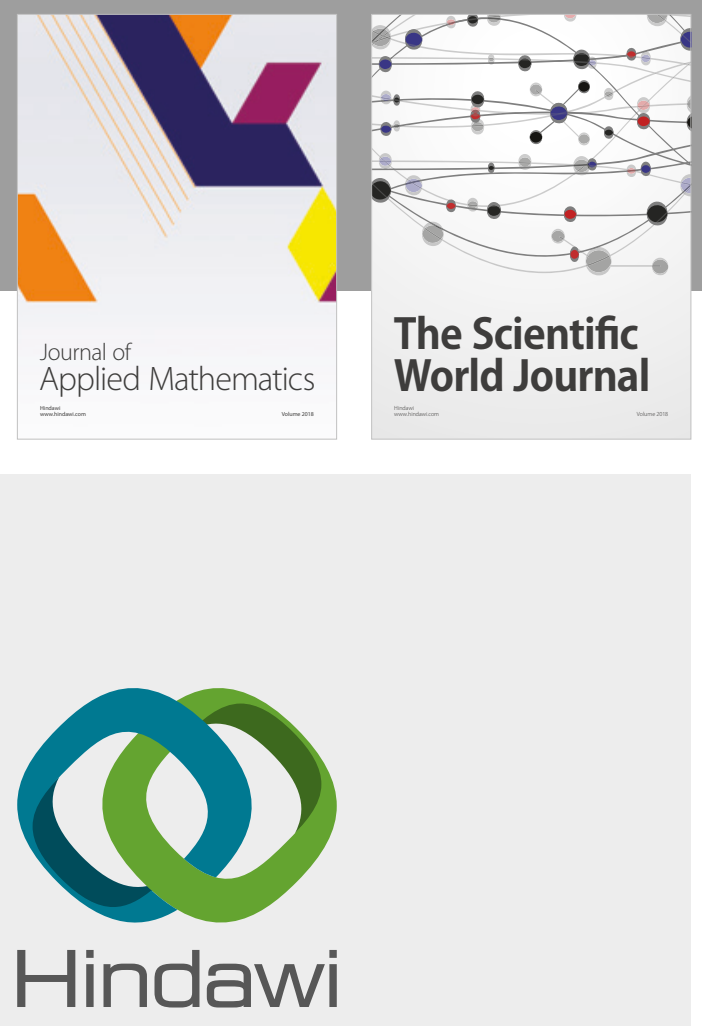

Submit your manuscripts at

www.hindawi.com

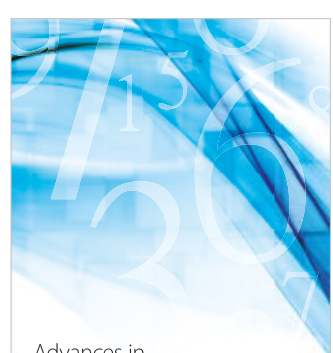

Advances in
Numerical Analysis
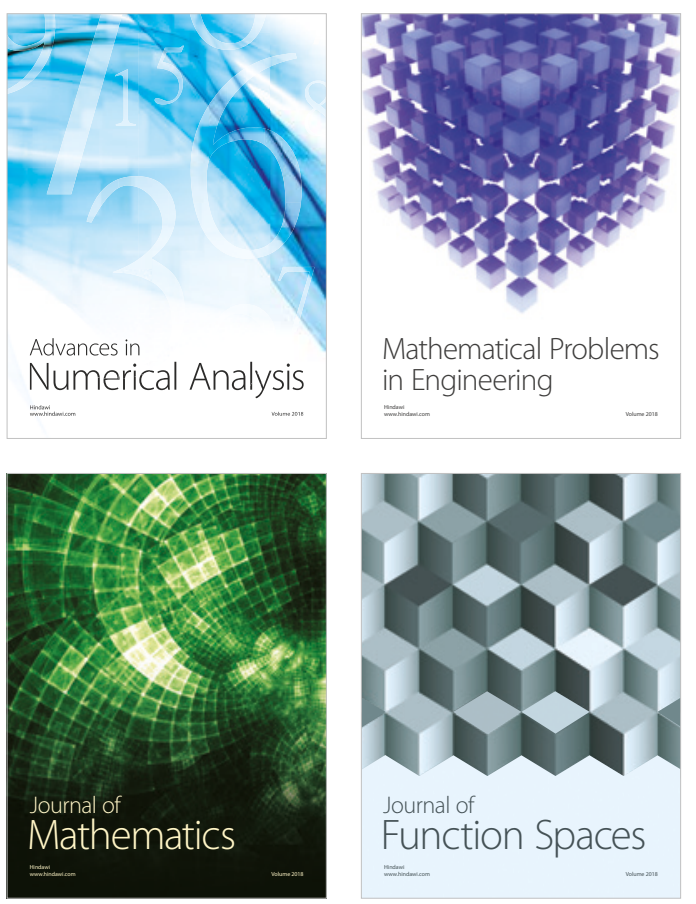

Mathematical Problems in Engineering

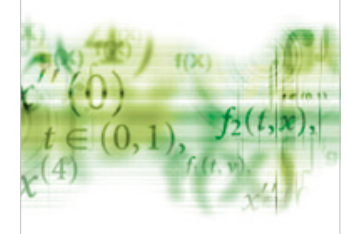

International Journal of

Differential Equations

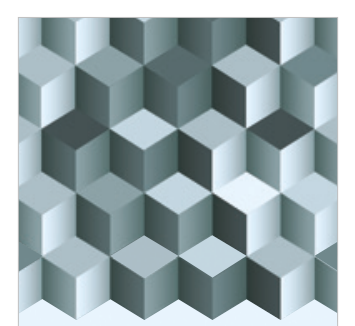

Journal of

Function Spaces

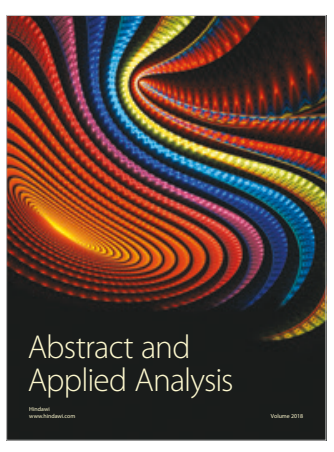

The Scientific

World Journal

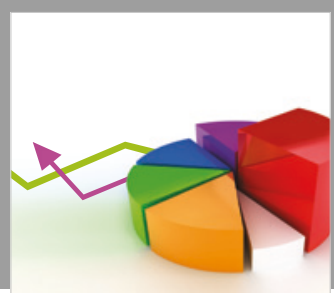

Journal of

Probability and Statistics
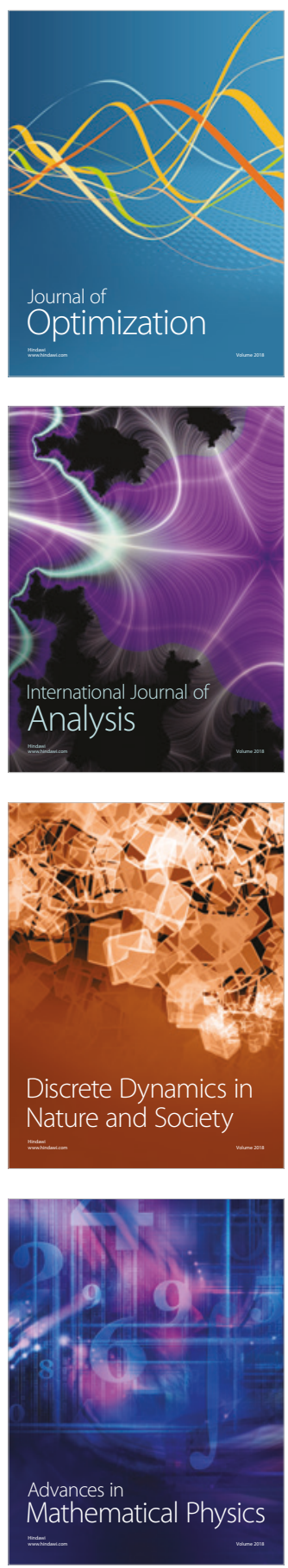